\title{
Engineering Design Knowledge Management in De-centralised Virtual Enterprises
}

Dr Hayder Aziz'1, Prof James Gao² and Prof Zhengxi Li 3

1i2, Dubai Media City, hayder.aziz@itsalat.com

${ }^{2}$ University of Greenwich j.gao@gre.ac.uk

3North China University of Technology, lzx@ncut.edu.cn

\begin{abstract}
The problems of collaborative engineering design and knowledge management at the conceptual stage in a network of dissimilar enterprises was investigated. This issue in engineering design is a result of the supply chain and virtual enterprise (VE) oriented industry that demands faster time to market and accurate cost/manufacturing analysis from conception. The solution consisted of a de-centralised super-peer net architecture to establish and maintain communications between enterprises in a VE. In the solution outlined below, the enterprises are able to share knowledge in a common format and nomenclature via the building-block shareable super-ontology that can be tailored on a project by project basis, whilst maintaining the common nomenclature of the 'super-ontology' eliminating knowledge interpretation issues. The two-tier architecture layout of the solution glues together the peerpeer and super-ontologies to form a coherent system for both internal and virtual enterprise knowledge management and product development.
\end{abstract}

Keywords: Virtual Enterprise, PLM, Ontology, Peer-to-Peer, STEP.

DOI: 10.3722 /cadaps.2008.xxx-yyy

\section{INTRODUCTION}

Collaborative engineering design and management in a large network of dis-similar enterprises has become a necessity in the new world of globalisation and outsourcing within the engineering industries. Formulating techniques and technologies in this area have been evaluated in depth [1]. In their review of collaborative conceptual design tools and techniques. Highlighting the gap that exists between the need for collaboration and the available tools which enable simple 'sharing' of design knowledge, the authors concluded that there is a major gap in the techniques to enable real time collaboration in a heterogeneous environment.

Previous research on the topic had studied various aspects of the problem including the Palo Alto Collaborative Testbed (PACT) by the Advanced Research Projects Agency (ARPA) where existing engineering tools were integrated using an encapsulating agent upon which a number of developments were developed by research teams including JATLite, a Java based agent infrastructure [2] and METAMORPH agent based architecture [3] all of which shared the same principle of creating agents integrated with the individual applications to handle the collaboration between tools. The collaboration aspects of Virtual Enterprises (VE) in manufacturing were coined [4] as the 'virtual corporation'. This was further evolved into the ideas presented by [5] as 'an architecture for the virtual enterprise' which laid out the logical sequences for the establishment, operation and dissolution of a VE. Further elaboration on by [6] where the basic features of a VE were laid out, and techniques utilising the Standard for the Exchange of Product models (STEP) standard and client-server web based internet for enabling a virtual collaboration. 
Other perspectives on VEs include [7], on the other hand [8] realised the inherently de-centralised nature of a VE and formulated processes for creating and managing the VEs without actually suggesting a technical implementation for VEs. Reid's formulation [8] for the inherent de-centralised nature of a virtual enterprise forms the foundation for [9] which developed the potential topology using a super-peer net architecture to overcome the limitations of a pure peer to peer system but without the structural and ownership issues arising from centralisation of knowledge.

Collaboration within the context of engineering design is the sharing of existing engineering design expertise between individuals from within a VE that is composed of entities that are collaborating in a one-off venture and have not collaborated previously. The entities are presumed to be dissimilar, meaning that they have a different internal company operation, product development process and tools to the other participants in the VE.

Engineering companies with a variety of Original Equipment Manufacturers (OEMs) customers face a huge problem with meeting the individual requirements of their clients. Interaction with client and product definition primarily takes place during the early concept stage of product development. Thus it is imperative that participants in the VE network (clients, engineers, and VE entities) can interact effectively and produce concept designs for the product that are practical to manufacture, cost effective and do not repeat the same mistakes made in earlier projects (this of course does not preclude the addition of new design and thought flaws).

Conceptual design is an essential part of the product lifecycle, which has a disproportionate effect on the costs, and eventual success, of the artefact. Traditionally this phase of the project is carried out in face to face meetings and brainstorming sessions with many delegates, from the different disciplines in the enterprise, with external customers and suppliers also taking part. Problem arises when enterprises wish to collaborate with a large number of suppliers and original equipment manufacturer (OEM) customers (by large number, this research assumes a many-many relationship). Current applications enable collaboration using business-to-business (B2B) protocols. However, these do not take into account that disparate enterprises do not have unified data models or workflows. This lack of standardisation results in a lot of time and money spent mapping B2B links between different vendor's systems as well as the uniquely customised data models and workflows implemented on top of vendor-specific applications. Within the setting of a Virtual Enterprise (VE), where alliances can include dozens of enterprises and often last for only a single project, these costs would be prohibitive (because it would in effect entail repeating the task of inter-enterprise integration between each enterprise collaborating in the VE). This is a strong factor in reducing the ability of large enterprises to participate in one-off VE partnerships, reducing agility and potentially losing business.

Some solutions have included the use of application service providers (ASP) or enabling a large enterprise's data system to function as an ASP for their collaborators' benefit in a VE setting. However the problem with this type of system is the management of intellectual property of the individual enterprises and whether enterprises can give up control of their information to a partner, who may, especially in the case of small and specialised enterprises, also be collaborating with their competitors simultaneously. Such fears can potentially hinder the formation of VEs between enterprises of any size.

Knowledge Management. The enterprises' prime asset, its knowledge, whilst stored in an electronic format, is not managed coherently thus perpetuating an 'invisible' limit on the company's knowledge, based on the impulse of knowledge workers' recollections of previous experience. Researchers and vendors have invariably attempted to solve the problem of coherency through centralisation; however this is not applicable in a collaborative and distributed development environment which is inherently de-centralised. The attempts at inter-enterprise collaboration are further complicated when enterprises with different depth of detail in their data models and different taxonomies (in this research, taxonomy is a term used to denote the data model, or models, used internally by an enterprise to manage its product and project data) collaborate together. Creating a situation in which detail is lost in the transfer from high to low detail enterprise data systems.

Finally, the prime reason for this investigation is that SMEs have up to now been left out of developments in PLM, knowledge management and VE developments in spite of forming the majority of the world's engineering community. This negatively affects the ability of SMEs to manage their knowledge, reuse existing expertise, collaborate with other SMEs in the virtual enterprise settings and collaborate with the larger enterprises that form their customer base. This weakness affects not only the SMEs but also the large enterprises that utilise SMEs within their supply chain or VE network.. 


\section{FORMAT AND MEDIUM OF INTER-ENTERPRISE COLLABORATION}

One of the foundations of effective collaboration is communication. Communication, within the context of this research is thought of as composed of a medium and a format. Once mediums and formats are formally established, comprehensible communication between two entities may take place. This is applicable to any type of communication. For example a conversation between two persons would be:

- Medium: Oral (face-face).

- Format: Language (known to both).

Or another example would be written communication between groups of people would be:

- Medium: paper and ink.

- Format: alphabetical characters in common language.

Naturally both these elements must match at all collaborating parties for mutually understandable communications to take place. This component of the research aimed to solve the key problem of establishing communication links between geographically separate and organisationally unrelated entities, rapidly and at low cost, with the aim of the communication being the collaborative development of engineering products. Whilst this may sound like a simple problem in today's world of standardised data-telecoms, the lack of end-to-end standardisation of taxonomies, authentication / access control systems and formats between enterprises has been a glaring shortcoming of modern Business to Business applications. In effect this has been one of the major drawbacks that has stunted the growth and spread of PLM and KM technologies to smaller enterprises today.

Figure 1 illustrates the holistic operation of the system including the heterogeneous ontologies, the medium to transport the data between the ontologies and the format for understanding the communication. Ontology for enterprise A contains the business and process knowledge for company "A" within the virtual enterprise. Similarly Ontology for enterprise"B" contains the domain ontology (which is in this instance DIFFERENT from the ontology in enterprise "A"). The "medium" in this figure is simply the means by which communications takes place (in this instance via TCP/IP i.e. over the internet) The red line denoting the format of the taxonomy denotes the file formats used to encode the ontologies of enterprises "A" and "B". Finally the workflow processes aim to glue the disparate business processes from the unrelated enterprises to enable the automation of the business processes in the VE.

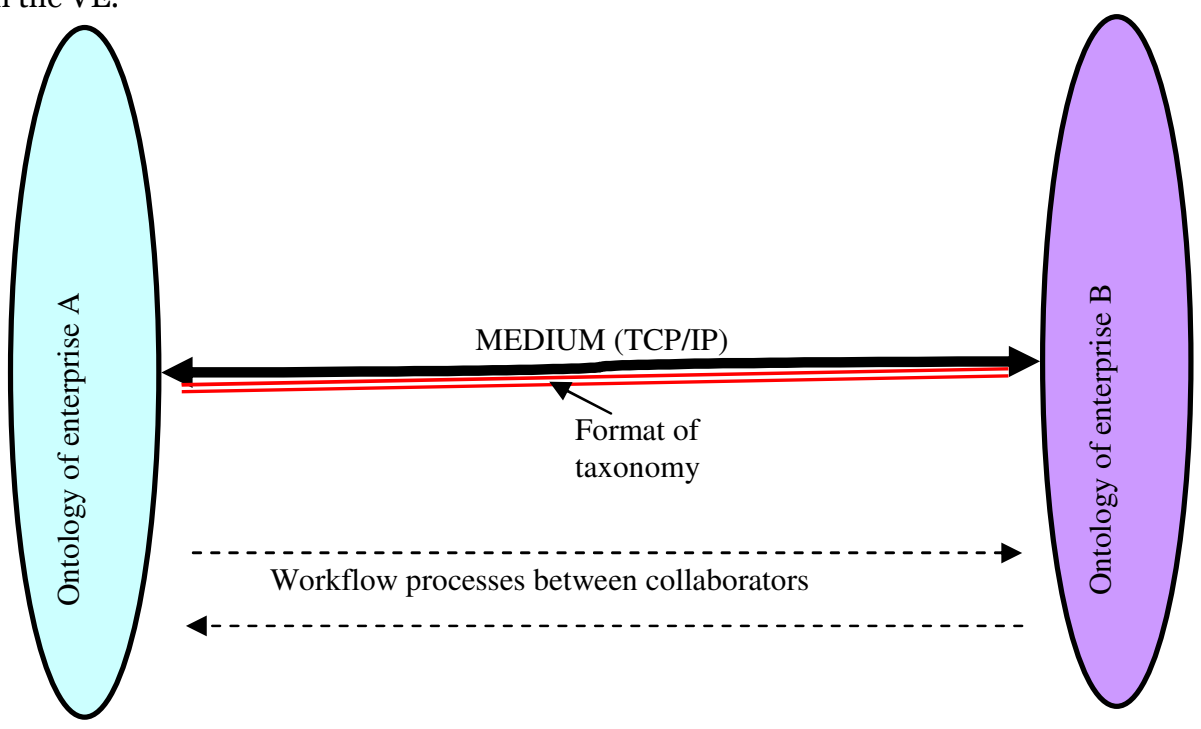

Fig. 1 Format, Medium, Ontology and workflow

The 'format' aspect of collaboration between enterprises is independent of the medium used. However the key problem, within the context of this research, of the format is creating a universal language and taxonomy (taxonomy in this context is the nomenclature used in a collaborative engineering setting) that will meet the following challenging criteria, which are a subset of the overall aim of the research:

- Providing a superset of all possible taxonomies in use today or in the future for inter-enterprise collaboration between engineering oriented entities. 
- Providing a format that can be used commonly by all and at no cost to enable the rapid dissemination and implementation by all parties in a collaborative venture.

- Enabling the rapid authentication and access management of all participants.

- $\quad$ Providing a method to rapidly resolve syntactic differences between different enterprises' data structures.

The above mentioned requirements have proven to be a tough challenge for researchers in the past as shown by the disparate technologies which only look at partially solving the problems. The solutions in existence today include well established International Standards (OSI IEEE 802) for network links and data communications protocols such as Transport Control Protocol/Internet Protocol (TCP/IP). However even though there are clearly established guidelines and even standardised formats (such as XML and RDF standards) there remains a practical disconnect in the sphere of standardised nomenclature (or taxonomies) for collaboration between disparate enterprises.

Since no two enterprises are the same, the idea of using XML based messaging for inter-enterprise collaboration is not easy as the collaborating companies' XML schemas have to be mapped to each other. In order to achieve speedy interoperability, a standard has to be set for basic messaging. There are a plethora of standards in development by various groups including RosettaNet, an endless list of XML schemas and data type definitions. These standards implement different levels of detail in the ontology. Some like STEP PDM attempt to implement the complete information structure of the engineering enterprise. Others like JuxtaPose (JXTA) implement only the "messaging" components.

The choice of standards to use depends on the level of "standardisation" that all enterprises can adhere to. However, with literally millions of enterprises, large and small, each with its own company-specific data structures and peculiarities, it can be safely assumed that radically altering the internal workings of all enterprises, to adhere to a single standardised taxonomy and data transfer medium is an improbable proposition on the grounds of the unsuitability of a standardised taxonomy for all companies' working needs and a lack of legislation forcing companies to adhere to any standardised medium.

To have a holistic overview of the problem, details of various company taxonomies and standards designed for inter-enterprise collaboration were collated. These are:

- $\quad$ ArvinMeritor taxonomy and data models (Tier-1 automotive supplier);

- $\quad$ ISO 9001:2000 management standards guidelines (simple management standard);

- ISO STEP-PDM (www.iso.ch), STEP standard taxonomy defining an internationally agreed upon taxonomy for engineering Product Data management, this standard complements the ISO-10303 STEP (below).

- $\quad$ ISO 10303 STEP, basic engineering product data, different Application Protocols define the data models for different industries. (see www.iso.ch for full details), generally modelled in EXPRESS, and stored in plain text format;

- $\quad$ RosettaNet - XML based generic B2B standardisation model;

- JXTA, (www.jxta.org) open-source generic low-level authentication and data management nomenclature for all types of applications (based on XML format);

Taxonomies above provided details of different characteristics of an enterprise's data model, and none of them can be considered to be a 'complete' representation of a company's data model (none of them claim to be, with the exception of SAP's massive taxonomies). The rest of the taxonomies however each provided a subset of overlapping functions that can be normalised to provide a 'standards based' enterprise data model usable by all. Excellent examples of taxonomies are the Application Protocol 2xx standards of the ISO-10303 STEP standard.

For the actual formats of the data, the following standards were looked at:

- XML - eXtensible mark-up language, a plain text format mark-up language used to define industry specific mark-up languages (some of which are defined above). BizTalk, MathML and RosettaNet are examples of taxonomies implemented with XML markup;

- OWL, Web Ontology Language is the definitive XML/RDF based ontology modelling and manipulation format incorporating Description Logics and constraints.

- EXPRESS is an ISO Standard (ISO 10303 Part 11) modelling and plain-text data-representation language for data models. (currently STEP, and STEP-PDM are defined using the express language and textformat P21);

Computer-Aided Design \& Applications, 5(1-4), 2008, xxx-yyy 
- SQL Structured Query Language, plain-text format relational database models and data definition language; and

- Proprietary CAD models with additional meta-data for non-geometric information.

\section{TWO TIER INTER-ENTERPRISE COLLABORATION ARCHITECTURE}

Figure 2 illustrates the problem of inter-enterprise data mapping and knowledge sharing graphically. The figure shows the data-model and data volume situation at present, where two fundamentally dissimilar data models from different enterprises (A and B) whose data models and depth of knowledge evolved over many years, attempt to establish a coherent Business to Business collaborative system between them. They are meant to illustrate the level of abstraction of the ontologies used within an enterprise vis-à-vis the ontologies shared between enterprises.

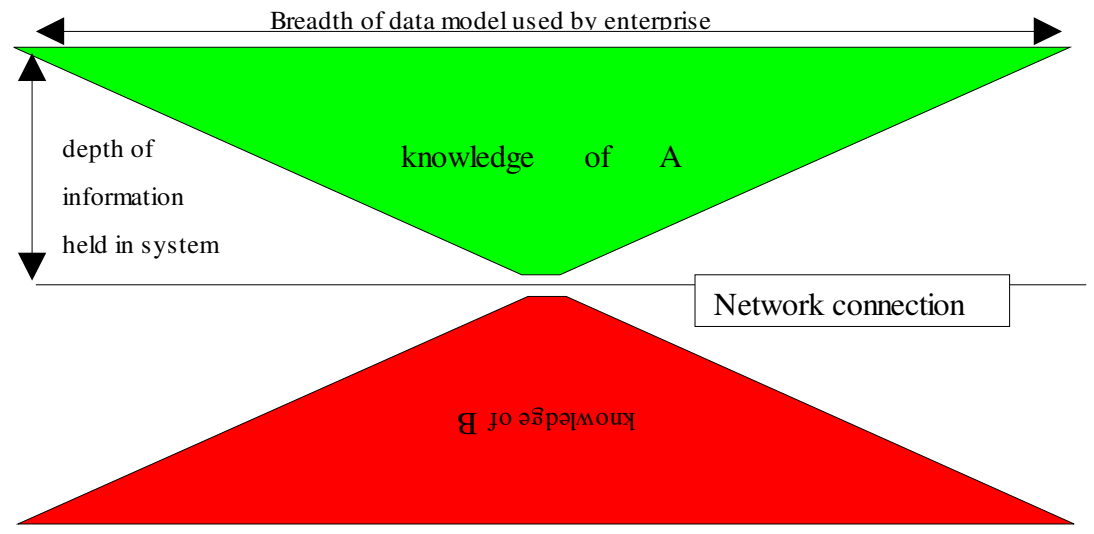

Fig. 2 Depth and breadth of traditional B2B connections

The layout of the diagram is designed to illustrate the complexity of the ontology (breadth), and the amount of knowledge that is actually stored in the knowledge base (depth).The 'area' bounded by the breadth of the ontology and depth of the knowledge constitutes the 'Knowledge to be shared' within the collaborative environment. It is practically impossible to match these data models on a one to one basis from one enterprise (e.g. A) to the other (e.g. B) rapidly, let alone on a one-to-many or many-many basis with multiple collaborating parties. With the above details in mind, in the view of the author only a very low level subset of all enterprises can be "standardised".

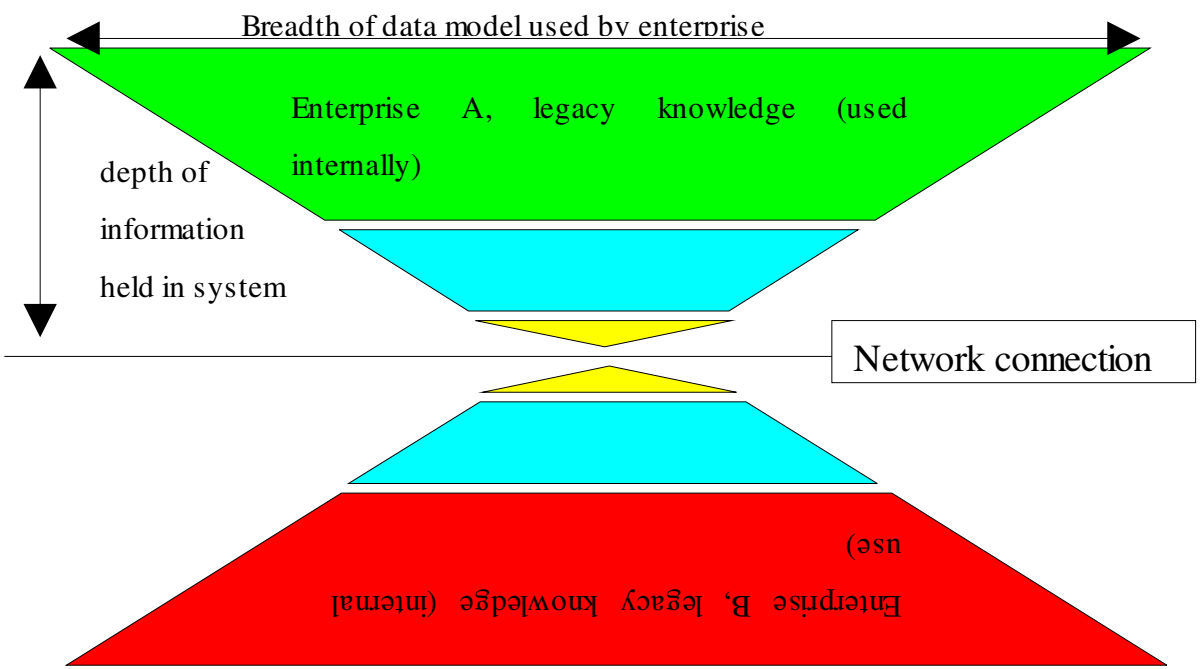

Figure 3 New B2B model with separate layer for authentication and establishing access-control 
This small subset should exist only as a medium to enable communication, identification and access control management (yellow tier, or tier-1 in Figure 4). All other aspects of the collaborative data environment are enterprise specific although constructed from a subset of ontological components as shown in (blue tier in Figure 4).

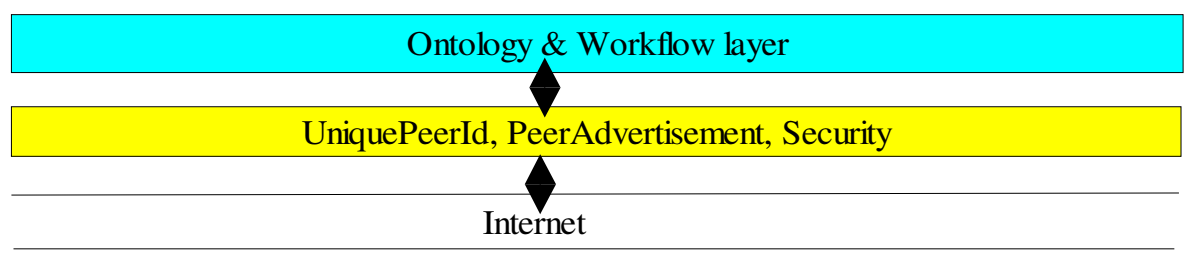

Figure 4 two tier communication architecture

This project created the two-tier solution as shown in Figure 3, which illustrates the overall architecture, and in Figure 4 which shows an exploded view of the light blue and yellow tiers of the architecture. The two-tier architecture is a unique idea which in effect solves one of the great problems of inter-enterprise collaboration, through a very simple yet effective solution. Each enterprise can customise and run its internal ontologies (Enterprise A in green and Enterprise B in red) and workflows within their own company's internal requirements, and when the company collaborates with another company, they can visually bridge the differences in taxonomies without technical complexities or programming. The communications and security aspects (authentication tier), see Figure 4 for a detailed breakdown, are handled automatically by the JXTA open protocol (the yellow tier). Speeding up of the integration process is further enhanced through the use of open standard semantic web enabled ontology formats (red and blue tiers) for storing the information, plus the use of open-standards in addition to company ontologies to define those ontology components. The blue tier bridges the gap between the internal and global ontology through the use of international standard nomenclature, the idea being that through the continued use of the blue tier, the depth of the red tier can be reduced (the depth illustrating the volume of knowledge held). The breadth of the red tier cannot however be eliminated as individual enterprises will always remain unique to an extent. However this system enables companies to persist as much of their reusable knowledge as is practicable in an open standard manner that enables rapid collaboration.

The aim of the system is to start off with a subset of the company's data models which are standardised for the requirements of a one off project, and the shared standardised data model to gradually permeate upstream into the company's existing base of knowledge and eventually supersede the internal data model after a number of collaborative projects actively increase the scope and depth of the new data model and thus rendering it an essential part of the company's daily practice. The reason the two-tier architecture can achieve such a critical mass of users are:

- The ontology and workflow layer implements a full subset of internationally agreed standards for product data modelling and business process automation;

- Because its a free easy to use package implementing acknowledged nomenclature standards in an open and extensible format, it can be used out of the box for collaboration by a large number of enterprises without hassle; and

- The flexibility and breadth of the data model is sufficient for supplanting and eventually replace legacy systems as its advantages permeate through the enterprise.

From above it can be seen that the critical aspect of the system is maintaining the ease of use and ease of extension that enables the collaborating partners and internal users to broaden the data model for their requirement within the scope of the ISO standards based on functional requirements and without the need for high level expertise. This is particularly critical for uptake in the small to medium sized sectors. These drivers were key to the idea of creating this flexible solution that accommodates the needs of established companies with internal knowledge bases, as well as the smaller collaborators with more limited requirements. The combination of the format and medium constitutes the first tier of the two tier architecture with the generic super-ontology constituting the second tier.

\section{DE-CENTRALISED VIRTUAL ENTERPRISE TOPOLOGY}

Peer to Peer ( $\mathrm{P} 2 \mathrm{P})$ applications address the needs of de-centralised organisations to collaborate and share knowledge regardless of geographical location. The principle of $\mathrm{P} 2 \mathrm{P}$ has been around for a long time, and is today implemented in a number of applications such as instant messaging and file-sharing (www.GNUtela.com). There

Computer-Aided Design \& Applications, 5(1-4), 2008, xxx-yyy 
are already a number of P2P PLM in existence including Alibre, a P2P CAD/PLM and collaboration tool in one. Figure 5 illustrates the proposed hybrid super-peer net architecture for inter-enterprise collaboration in egalitarian Virtual Enterprises. The yellow bubble shows an exploded view of the internal peer-net of the enterprise. Inside this internal-peer net there are a number of functional sub-systems as well as internal users. Each function within the super-peer net is a standard JXTA node (including the super-peer itself). The actual difference in the function of each node is down to the configuration selected for that node. This allows absolute flexibility for dynamically changing modifying adding and removing nodes within the internal peer net. It is also possible to make any one or many of the internal peer net nodes an external peer-net member via a simple configuration change. The functions used in this instance were the protégé knowledge base (this is physically colocated on the same machine in this example, but for the sake of topological accuracy has been included as a separate node), Each " $F$ " peer is an end user accessing the internal peer net (and the external super-peer net via the super peer). The PL node has the internal workflow functions of the peer net that are accessible by the wider super-peer network via the super-peer.

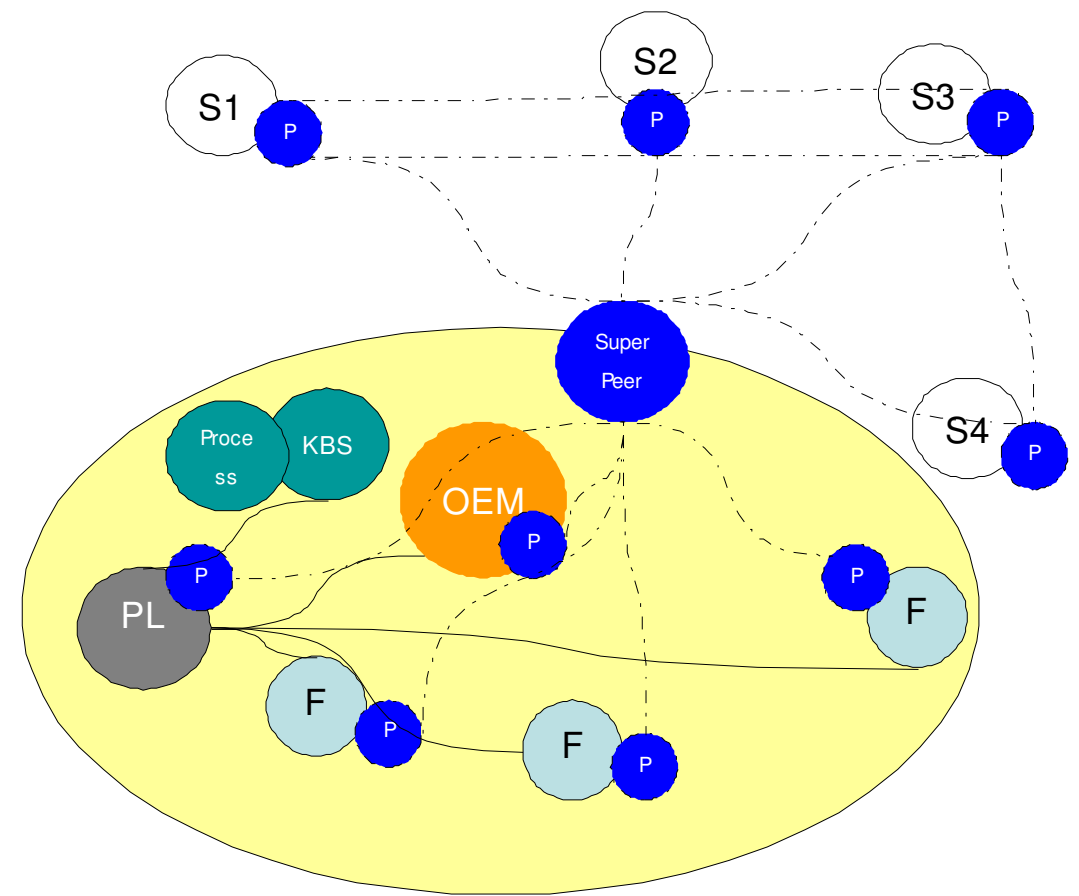

Figure 5 Super peer architecture showing the interaction within internal peer nets and interaction with external peers

Outside the peer-net is the external peer-to-peer environment which interlinks all the super-peers from every collaborator together. Note that each super-peer is connected to every other super-peer making a multiple redundant connection. In addition one, or many of the super peers within the network act as cache and rendezvous peers to enable peers or super-peers with intermittent connections to effectively participate in the peer network by having their internal shared knowledge bases stored on cache in case they are not available to serve other peer s that need to access their knowledge. This enables peers to connect to each other and query for and manipulate knowledge on different peers on the internal and wider peer-net transparently. The architecture was set-up with a super-peer net in order to create a number of virtual servers at each of the project participant's sites. These super peers are visible as peers to both the other super-peers and the internal peer net which they serve. They also act as the default rendezvous peer, and peer information is shared between the super-peers in order to improve the redundancy and query speed of the system.

\section{SUPER-ONTOLOGY FOR ENGINEERING KNOWLEDGE MANAGEMENT}

Once the mediums and formats used to establish communications between the collaborating partners was finished. there was thence a need to do something with this collaboration. The aim of the entire research was, to recap, help collaborative enterprises working in distributed and incoherent environments to work together rapidly 
and efficiently from the smallest participants to the large OEMs. The second major component of the research was to establish formal working methods and systems for persisting, managing and using the huge amounts of data and information generated and inferred in a typical product lifecycle (stored in the light blue shaded tier in Figure 3 ), and present and share it in a way that enables the collaborators to infer the correct interpretation and hence enhance the knowledge base of the collaborative project as well as the individual participants (Figure 4).

As mentioned earlier, in the author's opinion, the evolutionary, efficient storage of knowledge in easy to retrieve and easy-to-reuse formats reduces the amount of time re-learning and searching for existing knowledge and can be hoped to aid in the efficient and steady evolution of the wealth of knowledge persisted within the internal enterprise as well as the wider collaborative Virtual Enterprise. With this in mind, this section will go through a number of developments which were made to aid individual as well as collaborative enterprises in their internal and collaborative knowledge management (in this instance ArvinMeritor as test cases). The development of the common product development ontology is interrelated to the problem of collaboration and problems of collaborative design between enterprises of different sizes and complexity, that is, mapping between high and low content data models which results in irretrievable loss of information from the high content data model. This problem cannot be overcome traditionally by creating a mapping from one data model to another. Instead the author has sought to create a project oriented ontology that can be created, shared and used by all parties collaborating in an enterprise in real-time (in this instance an ontology is a fully defined taxonomy of a domain of knowledge).

The data gathered from the companies was organised hierarchically to obtain a basic structure of classes, subclasses and attributes, and then further re-organised into classes and slots (whilst eliminating duplicates). The company-specific sub-ontologies for ArvinMeritor were constructed. Normalisation of the data to eliminate duplication of information was followed by generalisation to standardise the nomenclature of information within the ontology. A knowledge base has been defined for geometry using the Protégé system as the User Interface and Java Expert System Shell (JESS) as the programming language. The complete design knowledge required for analysis is stored in the knowledge base in STEP Application Protocol (AP-224) for manufacturing features. The relationships and rules for AP-224 were originally defined in the EXPRESS language, some of the rules being rewritten using the Knowledge Interchange Format (KIF) format with a forward-chaining algorithms in JESS for faster model verification. For some of the model constraints the Protégé Axiom Language (PAL) was used, this is being used to construct queries for users.

\section{APPLICATION EXAMPLE}

Detailed below is the generic super-ontology component that was constructed. It is important to note at this point that the nature of the ontology that defines such a wide scope of a company's activities is by its very nature interlinked between different classes under different classifications. It is in fact possible to reach knowledge contained under 'requirement' from the 'bill of material' and to reach the customer_supplier_management from specifications. In further detail: Specifications: The specifications for the engineering design product are produced / derived from the Requirements information (which is itself based on the RFQ). The final class under specifications is 'Specification_items' which contains the variables that are used in the specification creation and management section (181 possible objects). The sub-sections of specifications deal with (in this example) latches for cars, and the nomenclature and structure reflects the specific requirement. The specifications themselves are split into multiple sub-specifications for safety, security, reliability, system integration, noise specifications, aesthetics specification, ease of operation and intuitive operation of the automotive latch (Figure 6).

Computer-Aided Design \& Applications, 5(1-4), 2008, xxx-yyy 


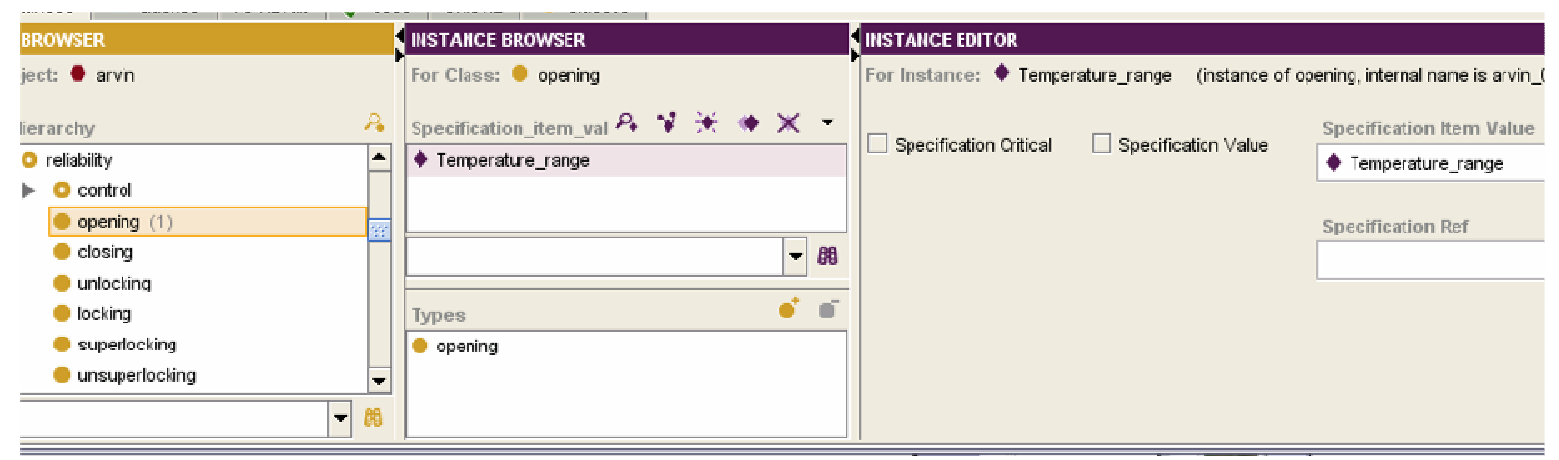

Figure 6 specifying the reliability of latch opening

The interface is split into three panes the left pane shows the top-level specification and sub-specification classes, the middle pane shows the "specification items" variables used in the specifications definitions and the right pane shows a description of the highlighted object from the middle pane. To illustrate the operation one example is shown below. The reliability required of the latch is specified. The user selects the 'opening' sub-section of the reliability section. In the centre pane the user can specify the opening reliability specification object (in this instance it is the temperature_range) which is a predefined standard object within specifications (obtained from the specification_items class, which defines the possible variables for specifications). The right pane is the data entry form where additional variables can be added and the value and level of importance for the 'temperature_range' variable within the opening reliability specification can be amended (there are default values from previous projects which are automatically actuated when first instantiating the variable in the specification construction to aid inexperienced users on the specification construction stage, which can be, of course changed from this form).

Figure 7 shows the latch component assembly of the example item being developed produced by the VE. These specifications, once constructed form the foundation of what the engineering design works towards. The real strength of course is that all the knowledge base specifications are defined in the same manner, enabling the design engineer to immediately bring up older projects with similar variables and reuse it in the new design.

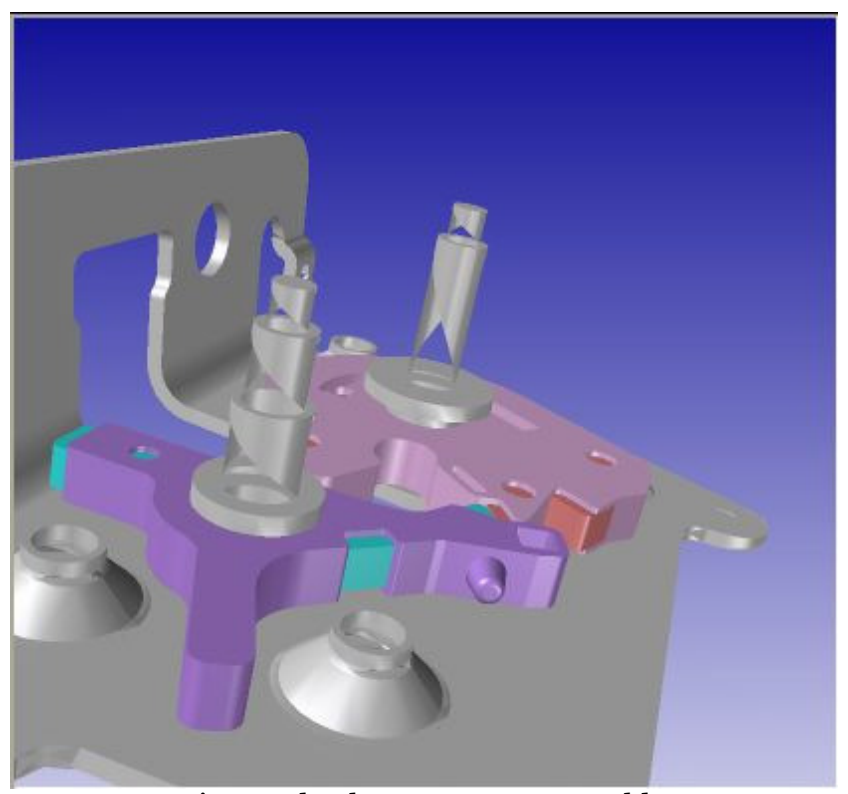

Figure 7 latch component assembly 
Figure 8 illustrates the actual location of the temperature range instance for the opening specifications of the reliability sub-class of the 'specification' definitions of the knowledge base. This demonstrates the level of depth of the ontology constructed and also serves to show the relative simplicity of the end user interface when taking into consideration the complexity of the actual ontology structure.

Bill of Material. One of the more complex components of the ontology is the Bill of Materials section. The figure below (Figure 9) illustrates the definition of the individual components of the bill of materials assemblies of all possible configurations within the knowledge base.

The actual structure of the Bill of Material component is a complex relationship between all the components of the system. Since the bill of material is the 'core' of the system it brings together the various VE participants (who make components of the final product') as well as the OEM who manages the specifications according to the requirements.

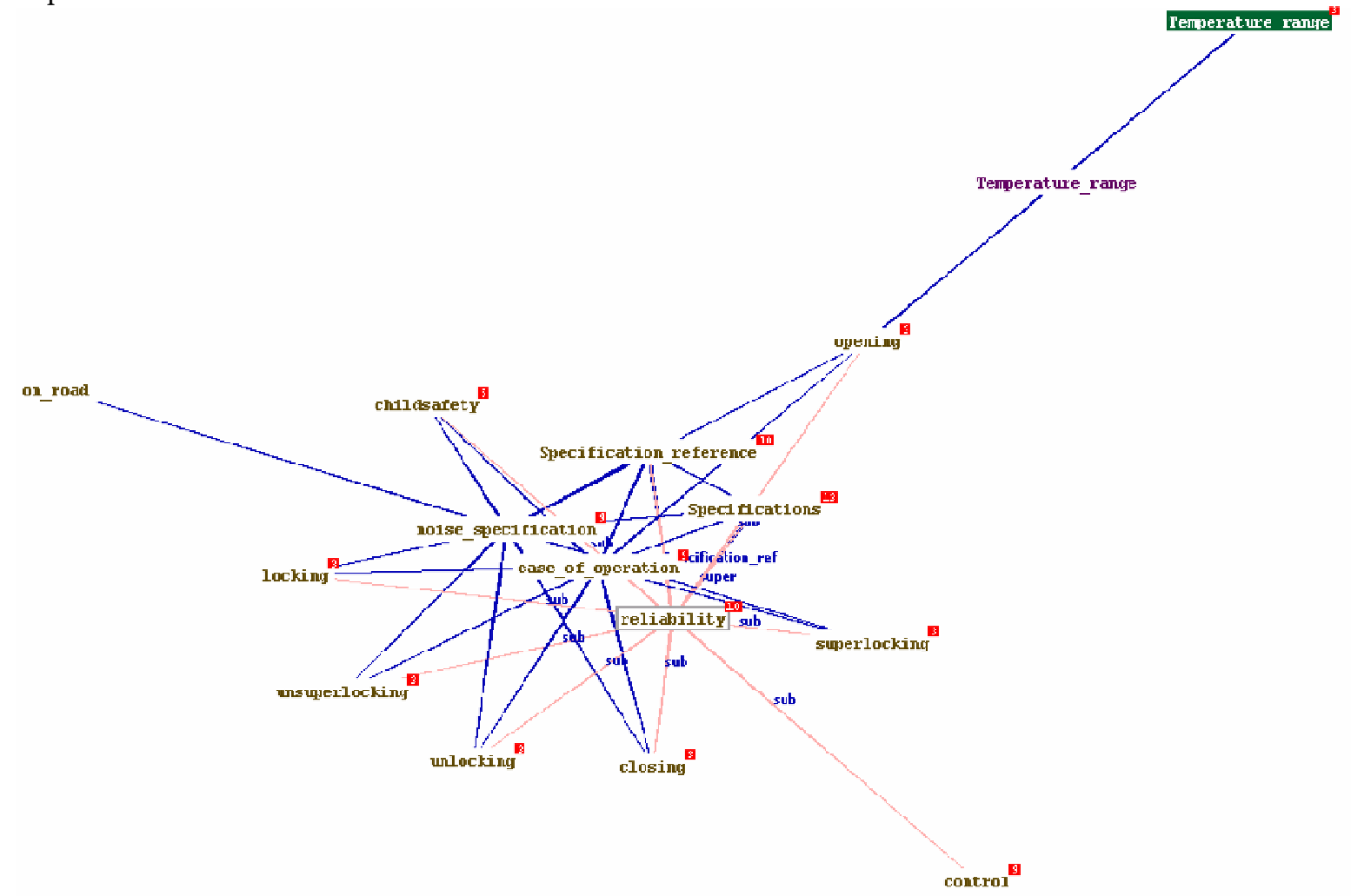

Figure 8 location of knowledge item for temperature range of the reliability of opening of latch

Computer-Aided Design \& Applications, 5(1-4), 2008, xxx-yyy 


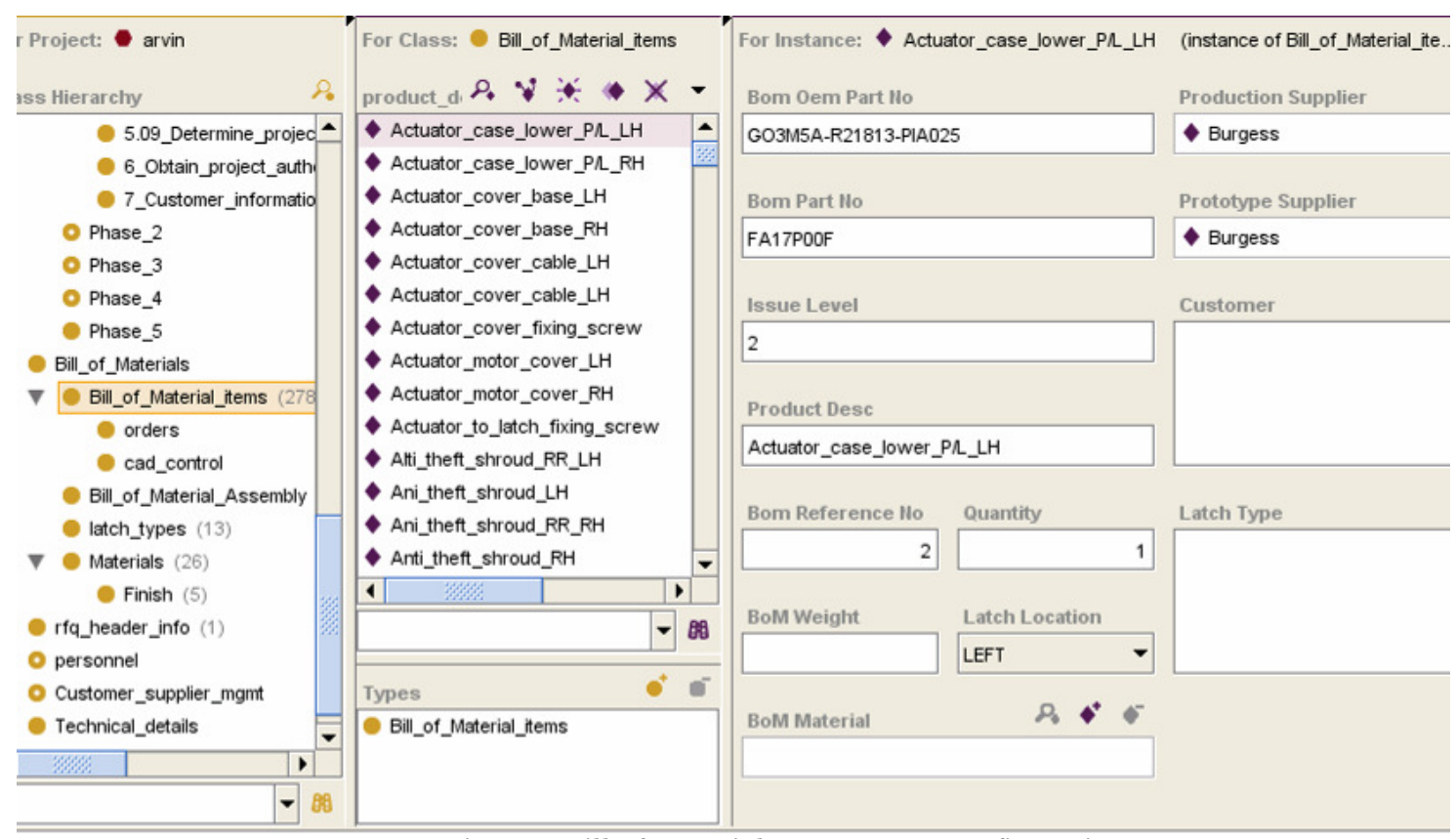

Figure 9 Bill of materials component configuration

An illustration of the internal structure is Figure 10. The final product is an instance of 'bill of material assembly and is composed of bill of material items. Each of the items has materials, suppliers, finish quality, configuration (latch type), orders and versions (cad_control) associated with it. A user adding a new component to the engineering design will fill in the component form in the bill of material items form that will automatically populate the rest of the knowledge base with new materials, new manufacturing processes and new latch type configurations (if applicable), this would then be available generally when creating new bills of materials for design Workflows. The workflows function as a quality management tool within an overall concept and product development process. These were implemented using the XPDL Open Workflow specification in order to keep the workflow implementation portable across many different enterprise applications. The actual workflows were simply a glue to bind together the various tools and functions within the product development process and the workflows illustrated below demonstrate the closed-loop cycles usable for engineering change and other closed loop control mechanisms.

Customer Supplier Management This component of the super-ontology exists primarily to glue together the complex chain of enterprises within the VE in terms of their competencies, existing supply agreements and interenterprise relationships. 


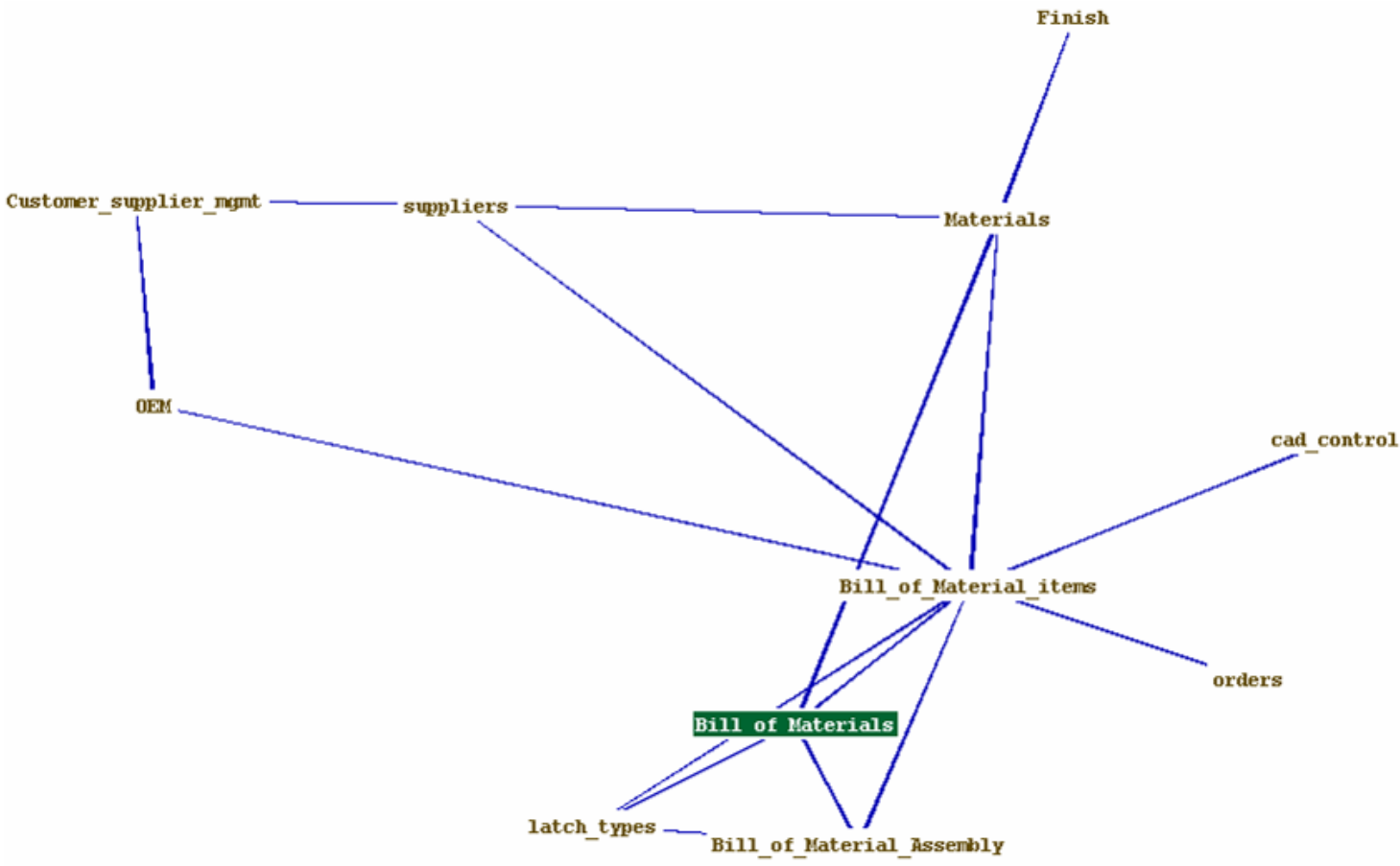

Figure 10 bill of material sub ontology structure

The information is automatically embedded and generated when a design iteration is created within the BoM. Figure 11 illustrates the complex inter-enterprise relationship in a simple diagram, from left to right a list of 'suppliers' or component manufacturers is shown. One of these 'Barton' is expanded to illustrate the relationship between 'supplier' a component (a claw_and_pawl_rivet for a latch), the associated material (BS3111_type_9/2_con_B), and via the customer supplier management module the relationship is explicitly defined with the 'OEM'.

Looking from right to left, the relationship is seen from the 'OEM' perspective. Here a top level OEM 'Volvo' is used as an example to illustrate the links in relationship between the enterprises in the VE. The backplate subassembly for the front right latch is used as the example. This is produced by the 'gammastamp' enterprise from the 'supplier' class of enterprises. Notably, the system also indicates that the company gammastamp is also the production supplier for another item 'claw and pawl rivet' that was used as an example of the item produced by 'Barton'. This illustrates a common situation within VEs where multiple enterprises are capable of producing the same components needed within the VE project. The system actively pushes such information to the VE participants enabling the pursuit of previously unknown potential producers based on the company's inherent internal ability + a project relationship with an OEM for a particular component. 


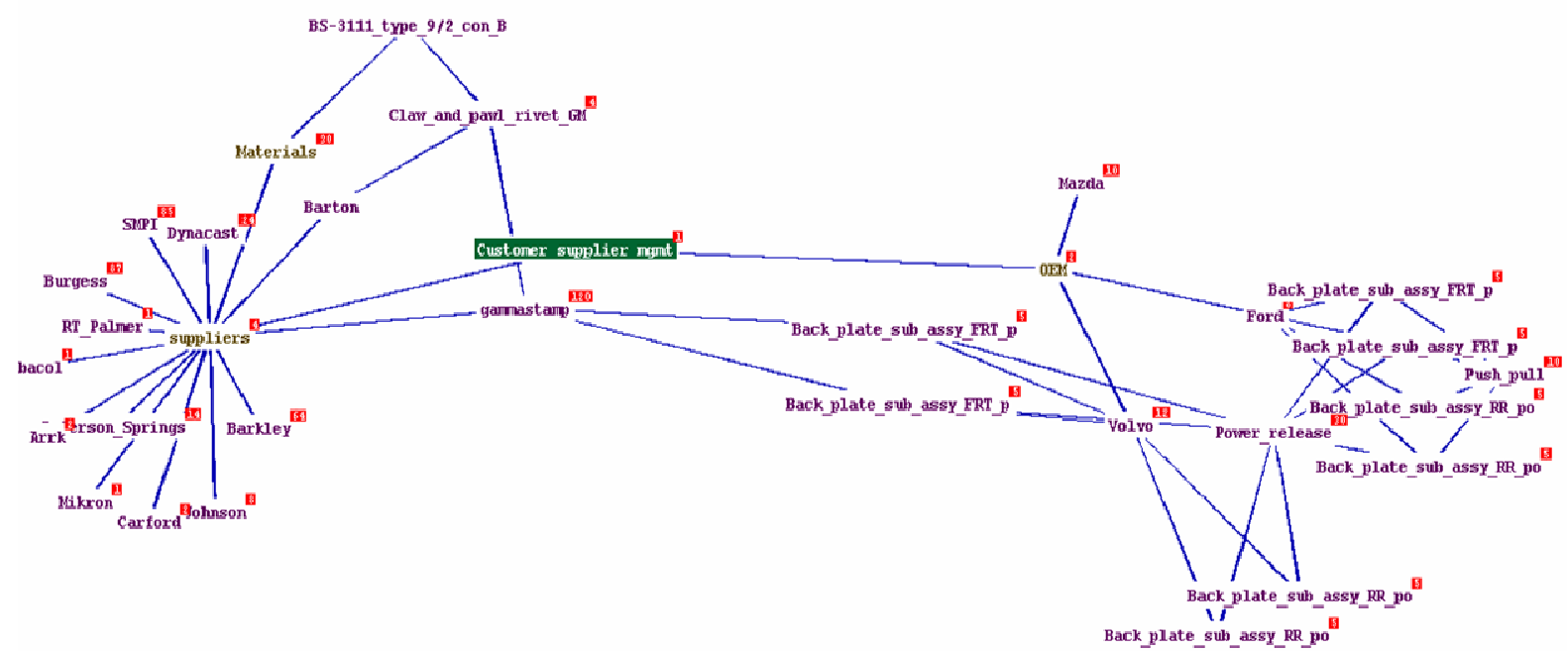

Figure 11 VE inter-enterprise relationship definition

The number of potential relationship webs is limited only by the number of enterprises in the chain and by the variety of products and materials they use. Here's another clearer view of the 'gammastamp' and 'Barton' production of the same item 'claw and pawl rivet' see Figure 12.

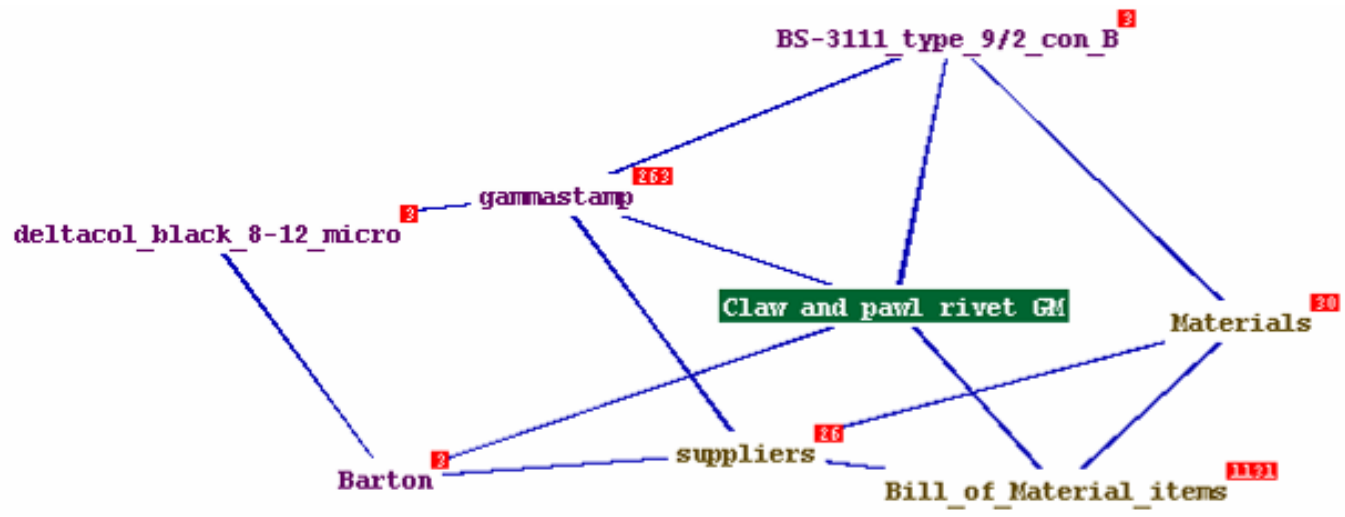

Figure 12 claw and pawl rivet relationships

\section{SUMMARY}

Overall results of the research have shown that large enterprise PLM systems are unsuitable for SME enterprises working in partnership with a large number of other small enterprises in a VE. Inherent centralisation, overly complex set up requiring many months for customisation, additionally the enterprise-specific customisation carried out cannot be reused on a system from another vendor. High end PLM systems have a sophisticated application layer for inter-enterprise communications, and the inherent weakness of this is the client/server paradigm whereby every enterprise needs to have custom integration between it and its extended enterprises' systems to function. The RosettaNet standard, can decrease the amount of customisation needed, but not eliminate it as RosettaNet only provides a subset of the messaging and data models of the inter-enterprise link. Finally, unlike the ontological 2-tier system, 
Other issues encountered were the difficulty in modifying the data models. PLM has a rather rigid data modelling interface based on the Unified Modelling Language (UML) to model the Java data model classes, and generate SQL scripts for the database. It can be modified by a professional who has to model, program, compile, update the database and integrate the code into the PLM before it can be used. This does not provide for the flexibility and ease of use for modelling ontologies that the project manager needs. The costs of implementation, licences and maintenance are very large by themselves and only practical when the enterprises involved can share the cost between each other over a long period of co-operation (as in an extended enterprise). However, the case with VEs, in which a partnership is valid for the relatively short period of a single project, this would be unacceptable. It had been discovered during the course of the project that centralisation, by its very nature, is an inhibiting factor for one-off inter-enterprise collaboration as the only methods available for collaboration in such an environment are B2B schema mapping integration, centralised web-enabled system or use of an Application Service Provider.

The use of domain specific ontologies distributed to the clients (users) via the $\mathrm{P}_{2} \mathrm{P}$ network and open standards: Elimination of the interoperability issues for product and project knowledge, enabling small enterprises to implement application standards that were beyond their means, such as STEP-PDM, and easier set up for interenterprise collaboration. RDF and OWL ontologies that can be queried by agent based systems and transformed by KIF based rules. The information contains the URI and meta-data eliminating the ambiguous context of the knowledge. Efficient query and retrieval mechanisms, intelligent agents to function on context aware information and provides an object oriented representation of an enterprise's data model.

De-centralisation: Elimination of centralised bottlenecks in bandwidth and resources, empowerment of collaborators within networks to "control" the knowledge they create, solving the management of intellectual property rights within VEs, elimination of centralised administration that results in reduction in cost and complexity and enable domain professionals to tailor the system. This, however, has a cost in terms of ease of maintenance and centralised control, as well as some query speed problems encountered due to the lack of a complete indexing service.

Comparison with other research It is impossible to make a direct correlation with the work of this research and that of other research in the field as each research focused on slightly different aspects of inter-enterprise collaboration and knowledge management and with different presumptions.

The work of $[4,5,6]$ laid out the foundations for defining the Virtual Enterprise in the earliest works on the topic. At that time, the telecom infrastructure available did not support the technical running of true VEs due to the high cost and low availability of bandwidth that would be needed to maintain Peer to Peer connections between multiple participants in a VE. This research is thus an implementation of some of the VE topology that the above mentioned research developed. Much of the detail created such as the super peer net architecture, and integration with ontology based KM systems were not discussed in the earlier research, and in that aspect this research explores the area of inter-enterprise interoperability in greater depth. Building on [10] the project took into account his observation that a true VE should function within the scope of a one-off project. Towards this end, this research constructed a 'super-ontology' usable between partners in real time and modifiable using the building block elements to eliminate entities not relevant to a particular project.

Comparing this work with some of the research works published on the topic of inter-enterprise integration also shows the overlap between this implementation and that of other researchers and also illustrates that different approaches to solving the problem of inter-enterprise integration are possible, depending largely on the types of enterprises collaborating, the nature of their collaboration and the hierarchy of the inter-enterprise relationship. [11] for example continues with the general trend of deploying a centralised system to manage inter-enterprise relationships (which is fine in a hierarchical relationship between the major enterprise and its 'collaborators'), of course this solution is particular to situations where there is a large controlling enterprise, and that is not the case in the scenario that this research is aimed at, the different scenarios of inter-enterprise collaboration being clearly illustrated by [12].

\section{REFERENCES}

[1] Wang, L.; Shen, W.; Xie, H.; Neelamkavil, J.; and Pardasani, A; Collaborative conceptual design \& state of the art and future trends , Computer-Aided Design , v 34,13 , 981--996 , (2002), Elsevier

[2] Cutkosky, M.R; Engelmore, R.S; Fikes, R.E; Genesereth, M.R; Gruber, T.R; Mark, W.S; Tenenbaum, J.M; and Weber, J.C; PACT: An Experiment in Integrating Concurrent Engineering Systems IEEE Computer, v $26,1,28--37,(1993)$

Computer-Aided Design \& Applications, 5(1-4), 2008, xxx-yyy 
[3] Shen, W; Maturana, F; and Norrie, D.H; MetaMorph II: an agent-based architecture for distributed intelligent design and manufacturing, Journal of Intelligent Manufacturing , v 11 , 3, 237--251, (2000), Springer

[4] Byrne, J. A; "The Virtual Corporation". (1993). Business Week. 98-103.

[5] Barnett, W; A. Presley, M. Johnson; and D. Liles (1994). "An Architecture for the Virtual Enterprise". In the proceedings of the IEEE International Conference on Systems, Man, and Cybernetics, San Antonio, TX

[6] Hardwick, M; Spooner, D.L; Rando, T; and Morris, KC; Sharing manufacturing information in virtual enterprises, Communications of the ACM , v 39, 2, 46--54, (1996), ACM Press New York, NY, USA

[7] Camarinha-Matos, L.M; and Afsarmanesh, H; The Virtual Enterprise Concept, Proceedings of the IFIP TC5 WG5. 3/PRODNET Working Conference on Infrastructures for Virtual Enterprises: Networking Industrial Enterprises , 3--14, 1999, Kluwer, BV Deventer.

[8] Reid, R.L; Rogers, KJ; Johnson, M.E; and Liles, D.H; Engineering the Virtual Enterprise, Automation \& Robotics Research Institute

[9] Aziz, H; Gao J; Open Standard, Open Source and Peer to Peer Tools and Methods for Collaborative Product Development. Journal of Computers in Industry, (2005), Vol. 56, 260-271.

[10] Beckett, R; Determining the anatomy of business systems for a virtual enterprise. Computers in Industry (2003), 51, 127-138.

[11] Ulieru, Mihaela; Norrie E Douglas; Kremer Rob; and Shen Weiming; A multi-resolution collaborative architecture for web-centric global manufacturing, Information Sciences Volume 127, Issues 1-2, August (2000), Pages 3-21

[12] Jagdev H. S; K.-D. Thoben; Anatomy of enterprise collaborations, Production Planning \& Control, Volume 12, Number 5 / July 1, 437 - 451, (2001) 